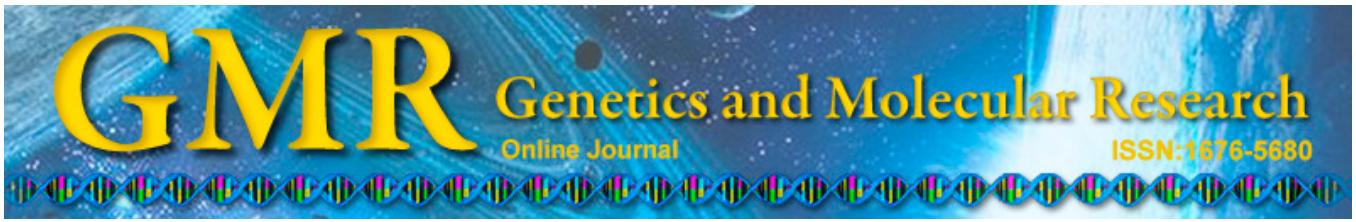

\title{
Gene expression profile analysis of testis and ovary of oriental river prawn, Macrobrachium nipponense, reveals candidate reproduction-related genes
}

\author{
H. Qiao, Y.W. Xiong, S.F. Jiang, H.T. Fu, S.M. Sun, S.B. Jin, Y.S. Gong \\ and W.Y. Zhang
}

Key Laboratory of Freshwater Fisheries and Germplasm Resources Utilization, Ministry of Agriculture, Freshwater Fisheries Research Center,

Chinese Academy of Fishery Sciences, Wuxi, China

Corresponding author: H.T. Fu

E-mail: fuht@ffrc.cn

Genet. Mol. Res. 14 (1): 2041-2054 (2015)

Received February 21, 2014

Accepted June 13, 2014

Published March 20, 2015

DOI http://dx.doi.org/10.4238/2015.March.20.14

\begin{abstract}
This study utilized high-throughput RNA sequencing technology to identify reproduction- and development-related genes of Macrobrachium nipponense by analyzing gene expression profiles of testis and ovary. More than 20 million 1 x 51-bp reads were obtained by Illumina sequencing, generating more than 7.7 and 11.7 million clean reads in the testis and ovary library, respectively. As a result, 10,018 unitags were supposed to be differentially expressed genes (DEGs) between ovary and testis. Compared to the ovary library, 4563 (45.5\%) of these DEGs exhibited at least 6-fold upregulated expression, while 5455 (54.5\%) DEGs exhibited at least 2-fold downregulated expression in the testis. The Gene Ontology (GO) enrichment analysis showed that $113 \mathrm{GO}$ terms had potential molecular functions in reproduction. The Kyoto Encyclopedia of Genes and Genomes results revealed that the most important pathways may be relevant to reproduction and
\end{abstract}


included 7 pathways. Forty-two genes were identified as reproduction-, development-, and sex-related genes based on GO classification and sequence comparison with other publications, including male reproductive-related LIM protein, spermatogenesis-associated protein, gametocyte-specific factor 1, VASA-like protein, vitellogenin, sexdetermining protein fem-1, and other potential candidates. These results will advance research in the field of molecular genetics in $M$. nipponense and offer a valuable resource for further research related to reproduction in crustaceans.

Key words: Reproduction-related genes; Differentially expressed genes; Testis; Ovary; Macrobrachium nipponense

\section{INTRODUCTION}

The oriental river prawn, Macrobrachium nipponense (Crustacea; Decapoda; Palaemonidae), is a commercial freshwater prawn species that is widely distributed in many Asian countries, including Japan, Korea, Vietnam, Myanmar, and China (Cai and Ng, 2002). Now, it has entered into a rapid development period and has become an important way to increase efficiency and income of agriculture with a total fishing production of 230,248 tons per year in China (Bureau of Fishery MoAPRC, 2011) and an annual production value of more than 100 million RMB. However, the oriental river prawn is the only species of freshwater crustaceans in large-scale production that can propagate themselves to overpopulation, especially in autumn (known as autumn-propagation). This causes a multi-generation reunion, increased breeding density, and risk of lack of oxygen. As a result, the autumn-propagation has become a problem in cultured stocks and restrained the health development of prawn aquaculture, which leads to low market value of the product because of low growth rate, poor survival, and short life span. Therefore, it is of great practical significance to study the regulatory mechanism of reproduction in oriental river prawn, which would help to delay early sexual maturity, regulate the reproduction rate, and improve the production benefit.

Various factors can influence the reproduction of crustaceans. Previous studies showed that some hormones produced by special glands, such as methyl farnesoate, gonad-inhibiting hormone (GIH), and mandibular organ-inhibiting hormone, can obviously stimulate or inhibit gonad development (Somka et al., 1993; Wainwright et al., 1996; Olmstead and Leblanc, 2002). Removing these special glands, such as the androgenic gland, can also be effective by promoting sex-reversed populations (Sagi et al., 1990). Some other exogenous factors, such as sex hormones (17 $\beta$-estradiol and progesterone) and environmental factors (temperature, light, and feed conditions), were proven to affect gonad development in crustaceans (Sasser and Singhas, 1992; Pascual et al., 1998). These methods, rather than genetics, were not applied well in practical production. In recent studies, researchers expected to reveal the regulatory mechanism of gonadal development of crustaceans by constructing cDNA expression libraries and discovering reproduction- and gonad development-related genes (Krungkasem et al., 2002; Qiu et al., 2008a,b; Treerattrakool et al., 2008; Wu et al., 2009; Xie et al., 2010; $\mathrm{Ma}$ et al., 2012). For oriental river prawn, there were only two expressed sequence tag (EST) cDNA libraries (testis and ovary) with a total of 81,411 EST records in the National Center for Biotechnology Information (NCBI) database and 1 transcriptome (several tissues) reported; 
several genes ( $U b c 9$, gustavus, and kazal-type protease inhibitors) were reported to play an important role in gonad development in crustaceans (Wu et al., 2009; Qian et al., 2012; Qiao et al., 2012; Ma et al., 2012). However, tissue-specific gene expression profiles in gonad of $M$. nipponense are not currently available.

Recent research has shown that high-throughput RNA-sequencing (RNA-Seq) technology is a powerful, cost-efficient tool for gene expression profiling analysis and provides a more sensitive tool than microarray methods (Marioni et al., 2008). In this study, we investigated the gene expression profiles of testis and ovary of oriental river prawn. We aimed 1) to develop a gene expression profile database of the testis and ovary of oriental river prawn to identify candidate genes that are involved in reproduction and differ in expression between males and females and 2) to gain a global view of the molecular mechanism (s) whereby gene expression may influence reproduction and gonad development in oriental river prawn.

\section{MATERIAL AND METHODS}

\section{Prawn and tissue preparations}

Healthy adult male and female $M$. nipponense weighing 2.10-4.85 g were captured from Tai Lake in Wuxi City, Jiangsu Province, China $\left(120^{\circ} 13^{\prime} 44^{\prime \prime} \mathrm{E}, 31^{\circ} 28^{\prime} 22^{\prime \prime} \mathrm{N}\right)$. Male and female prawns were transferred to two $300-\mathrm{L}$ tanks (20 prawns in each tank) and maintained in aerated freshwater at room temperature $\left(20^{\circ} \mathrm{C}\right)$ for $72 \mathrm{~h}$ before sample collection. No prawns died during the period. After the period, 6 prawns from each tank were randomly selected and anesthetized under MS222 anesthesia to minimize suffering. The testes and ovaries from 6 individuals were collected and frozen in liquid nitrogen immediately until used for RNA extraction.

\section{RNA extraction and Illumina sequencing}

The differentially expressed gene (DEG) libraries (testis and ovary) were constructed as transcriptome libraries and an ovary library was constructed as a control library. Six male and 6 female prawns were dissected to obtain samples of testis and ovary. Total RNA was extracted using TRIzol ${ }^{\circledR}$ Reagent (Invitrogen, USA) according to the manufacturer protocol and treated with DNaseI. RNA integrity was confirmed by the 2100 Bioanalyzer (Agilent Technologies, Germany). The samples were prepared using the Illumina kit following the manufacturer recommendations. Two biological replicates were sequenced and analyzed. Two-strand cDNA libraries were prepared, and samples were sequenced on an Illumina HiSeq 2000 with a 51-bp single-end read length (GATC Biotech, Germany).

\section{Sequencing and functional analyses}

Clean reads were obtained by filtering the adaptor sequences and removing lowquality sequences (containing ambiguous bases). Then, the clean reads were mapped to the reference genome and genes of $M$. nipponense available at http://www.ncbi.nlm.nih.gov/ sra/?term=SRA051767 and nucleotide basic local alignment search tool (BLASTn), allowing no more than 2 nucleotide mismatches. Only the tags with a perfect match or 1 mismatch were further considered and annotated based on the reference genes. 
A statistical analysis of the frequency of each read in the different cDNA libraries was performed to compare gene expression in different tissues. The gene expression level was calculated using the reads per kb per million reads (RPKM) (Mortazavi et al., 2008) method. Both libraries of clean reads were normalized to the RPKM value to obtain the normalized gene expression level. A strict algorithm (Audic and Claverie, 1997) was performed to identify DEGs. The relative-change threshold in comparisons was performed using the absolute value of $\log 2$ (Testis$\mathrm{RPKM} /$ Ovary-RPKM). The threshold with a false discovery rate $(\mathrm{FDR}) \leq 0.001$ and the absolute value of $\log \mathrm{FC}$ ratio $\geq 2$ were used to judge the significance of gene expression differences.

Gene Ontology (GO) and pathway annotation and enrichment analyses were based on the NCBI Gene Ontology database (http://www.geneontology.org/) and the Kyoto Encyclopedia of Genes and Genomes (KEGG) pathways (http://www.genome.jp/kegg/), respectively.

\section{Quantitative real-time polymerase chain reaction (qRT-PCR) analysis}

To verify the DEGs, qRT-PCR was used to investigate expression levels. The qRTPCR analysis was carried out using SYBR Green qRT-PCR analysis in a CFX96TM RealTime System (Bio-Rad, USA). The protocol was performed as follows: a 96-well plate with a $20 \mu \mathrm{L}$ reaction volume containing $10 \mu \mathrm{L} 2$ X SYBR Green Premix Ex Taq (TaKaRa, Japan), $0.4 \mu \mathrm{L} 2 \mu \mathrm{M}$ of each primer, $2 \mu \mathrm{L}$ template, and $7.2 \mu \mathrm{L}$ PCR-grade water. The thermal profile for SYBR Green qRT-PCR was $95^{\circ} \mathrm{C}$ for $10 \mathrm{~min}$, followed by 40 cycles of $95^{\circ} \mathrm{C}$ for $15 \mathrm{~s}$ and $60^{\circ} \mathrm{C}$ for $1 \mathrm{~min}$. Diethylpyrocarbonate water was used in place of template as a negative control. A relative standard curve was constructed using 10 -fold serially diluted cDNA. Each sample was run in triplicate along with the internal control gene, $\beta$-actin. To ensure that only 1 PCR product was amplified and detected, dissociation curve analysis of amplification products was performed at the end of each PCR. The relative copy number of mRNA was calculated according to the $2^{-\Delta \Delta C t}$ comparative cycle threshold method (Livak and Schmittgen, 2001).

\section{RESULTS}

\section{Sequence read processing and mapping}

The Illumina HiSeq 2000 platform was used to perform high-throughput sequence analysis on the testis and ovary libraries to investigate the DEGs in gonad. More than 12.5 million single reads of $51 \mathrm{bp}$ were obtained from testis, and over $92.6 \%$ of the total reads were identified as clean reads before mapping them to the reference database. Over 7.7 million clean reads $(66.40 \%)$ in the testis library were successfully mapped to the reference database. In the ovary library, 15.2 million reads were generated, and more than $93.1 \%$ of them were clean. Among the clean reads, $83.07 \%$ were mapped to the database perfectly (Table 1 ).

\begin{tabular}{lcc}
\multicolumn{2}{c}{ Table 1. Major characteristics of the testis and ovary libraries. } & Ovary \\
\hline Name & Testis & $775,342,290$ \\
\hline Total nucleotides (nt) & $640,341,006$ & $15,202,790$ \\
Total reads & $12,555,706$ & $14,167,160$ \\
Clean reads & $11,631,094$ & $93.19 \%$ \\
\% Total clean reads & $92.64 \%$ & $11,768,079$ \\
Mapped reads & $7,723,096$ & $83.07 \%$ \\
\% Total reads mapped & $66.40 \%$ & \\
\hline
\end{tabular}


To discover the genes displaying a significant difference in expression in testis and ovary, the gene expression levels were calculated using the RPKM method (Mortazavi et al., 2008). A total of 81,397 entities were detected between the testis and ovary libraries. Using FDR $\leq 0.001$ and the absolute value of the $\operatorname{logFC}$ ratio $\geq 2$, we identified 10,018 significant DEGs that included 19,750 upregulated and 18,669 downregulated genes (Table 2). Compared to the ovary library, 4563 (45.5\%) of these DEGs exhibited at least 6-fold upregulated expression, while 5455 (54.5\%) DEGs exhibited at least 2-fold downregulated expression in the testis. The DEGs are shown in Figure 1.

Table 2. Differentially expressed genes.

\begin{tabular}{lcccc}
\hline & Total & Upregulated & Downregulated & Significant* $^{*}$ \\
\hline Testis $v s$ Ovary & 81,397 & 19,750 & 18,669 & 10,018 \\
\hline
\end{tabular}

*Differentially expressed genes (DEGs).

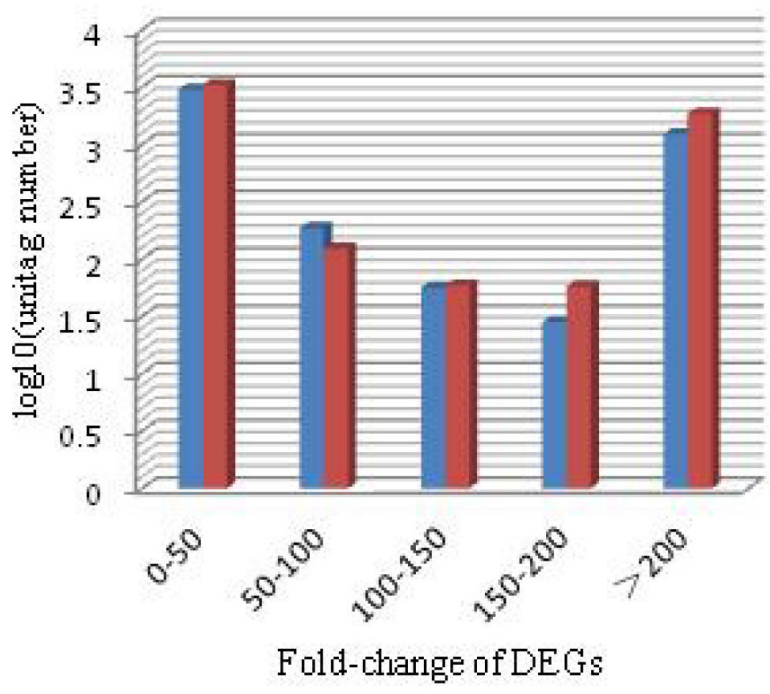

- Upregulated DEGs

Downregulated DEGs:

Figure 1. Differentially expressed genes (DEGs). The x-axis represents the fold-change of expression. The y-axis represents the number of DEGs $(\log 10)$.

\section{GO function analysis}

In GO analysis, 51,404 unique tags from M. nipponense were clustered based on matches with sequences with known function (Figure 2). These were divided into 3 functional categories: cellular component $(16,618)$, molecular function $(10,645)$, and biological process $(24,141)$. Most of the corresponding biological process genes were involved in cellular processes $(4663,19.3 \%)$. Most of the cellular component genes encoded proteins associated with cells and cell parts $(3697,22.2 \%)$, and most of the molecular function genes were associated with binding $(4609,43.3 \%)$. The GO enrichment analysis showed that $113 \mathrm{GO}$ terms had potential molecular functions in reproduction (Figure 3). The top $3 \mathrm{GO}$ functions were estab- 
lishment of localization $(\mathrm{P}=3.08 \mathrm{E}-10)$, small molecule metabolic process $(\mathrm{P}=3.43 \mathrm{E}-10)$, and transport $(\mathrm{P}=3.59 \mathrm{E}-10)$.

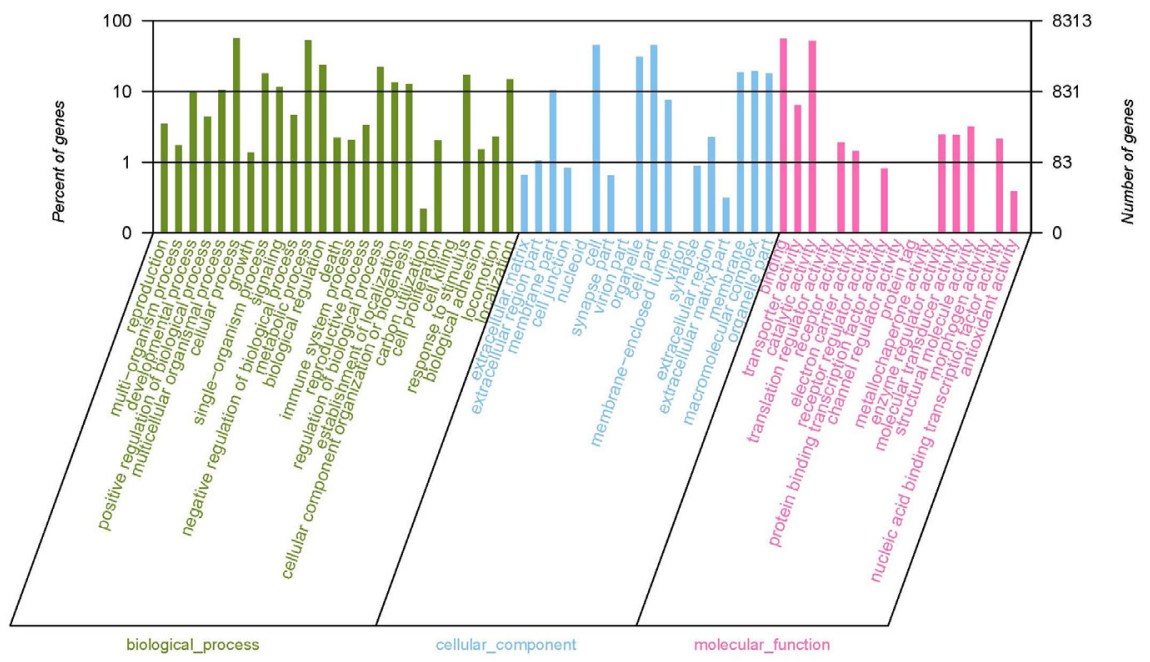

Figure 2. Distribution of gene ontology (GO) classifications.

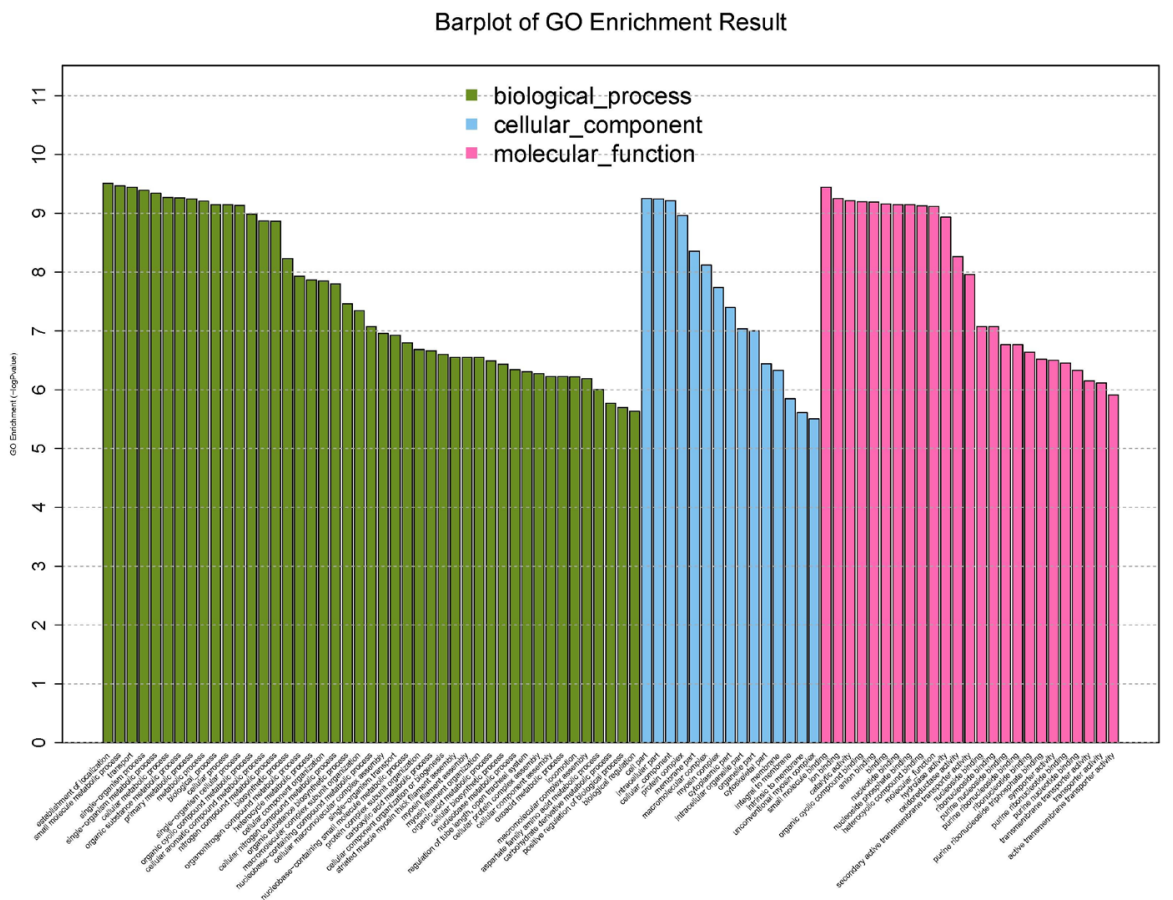

Figure 3. Gene ontology (GO) enrichment classification of differentially expressed genes (DEGs) from ovary and testis in Macrobrachium nipponense. 


\section{KEGG pathway analysis}

In order to determine the functional annotation of the large lists of DEGs, significantly enriched metabolic pathways and signal transduction pathways were identified. A total of 251 predicted KEGG metabolic pathways were associated with 2729 DEGs. Many of the pathways, including progesterone-mediated oocyte maturation, oocyte meiosis, MAPK signaling pathway, and ubiquitin-mediated proteolysis, might be related to reproduction and gonad development. Table 3 lists the top 25 pathways with the highest tag numbers. Pathways with $\mathrm{P}$ $<0.05$ were significantly enriched. Our results revealed that the most important pathways that may be relevant to spermatogenesis or reproduction included 7 pathways (Figure 4). They included metabolism of xenobiotics by cytochrome $\mathrm{P} 450(\mathrm{P}=1.14 \mathrm{E}-04)$; drug metabolism by cytochrome $\mathrm{P} 450(\mathrm{P}=2.61 \mathrm{E}-04)$; glycine, serine, and threonine metabolism $(\mathrm{P}=7.52 \mathrm{E}-04)$; retinol metabolism $(\mathrm{P}=1.38 \mathrm{E}-03)$; viral myocarditis $(\mathrm{P}=5.62 \mathrm{E}-03)$; naphthalene degradation $(\mathrm{P}=0.024)$; and tight junction $(\mathrm{P}=0.048)$. These predicted pathways are likely to be useful in future investigations focusing on their functions in M. nipponense.

Table 3. Top 25 pathways with the highest tag numbers.

\begin{tabular}{|c|c|c|c|}
\hline No. & Pathway terms & No. of tags & Pathway ID \\
\hline 1 & Lysosome & 52 & ko04142 \\
\hline 2 & Phagosome & 50 & ko04145 \\
\hline 3 & Tight junction & 45 & ko04530 \\
\hline 4 & Protein processing in endoplasmic reticulum & 41 & ko04141 \\
\hline 5 & Alzheimer's disease & 41 & ko05010 \\
\hline 6 & Focal adhesion & 37 & ko04510 \\
\hline 7 & Insulin signaling pathway & 36 & ko04910 \\
\hline 8 & Endocytosis & 36 & ko04144 \\
\hline 9 & Pathways in cancer & 36 & ko05200 \\
\hline 10 & Huntington's disease & 35 & ko05016 \\
\hline 11 & Viral myocarditis & 34 & ko05416 \\
\hline 12 & Regulation of actin cytoskeleton & 34 & ko04810 \\
\hline 13 & Oxidative phosphorylation & 34 & ko00190 \\
\hline 14 & HTLV-I infection & 34 & ko05166 \\
\hline 15 & Purine metabolism & 33 & ko00230 \\
\hline 16 & Spliceosome & 33 & ko03040 \\
\hline 17 & Pathogenic Escherichia coli infection & 32 & ko05130 \\
\hline 18 & Influenza A & 30 & ko05164 \\
\hline 19 & Vibrio cholerae infection & 29 & ko05110 \\
\hline 20 & RNA transport & 29 & ko03013 \\
\hline 21 & Oocyte meiosis & 28 & ko04114 \\
\hline 22 & Amino sugar and nucleotide sugar metabolism & 25 & ko00520 \\
\hline 23 & Parkinson's disease & 25 & ko05012 \\
\hline 24 & Ubiquitin-mediated proteolysis & 25 & ko04120 \\
\hline 25 & Glycine, serine, and threonine metabolism & 24 & ko00260 \\
\hline
\end{tabular}

ID represents the pathway ID in the Kyoto Encyclopedia of Genes and Genomes (KEGG). The pathway term represents the pathway name.

\section{Candidate reproduction-related genes and $q R T-P C R$ validation}

All 10,018 DEGs were subjected to BLASTn using the NCBI nr/nt database, and 3061 were assigned to known sequences in the databases. In other word, a total of $6957(69.4 \%)$ DEGs were novel. According to our results and published reports, many DEGs involved in reproduction in M. nipponense, including male reproductive-related LIM protein, sperm gelatinase, cathepsin D, gametocyte-specific factor 1, VASA-like protein, vitellogenin, and other 
potential candidates, were identified. Table 4 lists 42 DEGs that are related to reproduction, development, and sex selection from the expression profile. qRT-PCR analysis was used to validate the Solexa expression profiles. Six genes (spermatogenesis-associated protein 13, kunitz-type protease inhibitor 1, arginine kinase 1, vitellogenin receptor, male reproductionrelated protein Mar-Mrr, and extra sex combs) were randomly selected for qRT-PCR validation (Figure 5). qRT-PCR analysis of independent samples of testis and ovary tissues confirmed the results obtained by sequencing. The primers used in qRT-PCR validation are listed in Table 5.

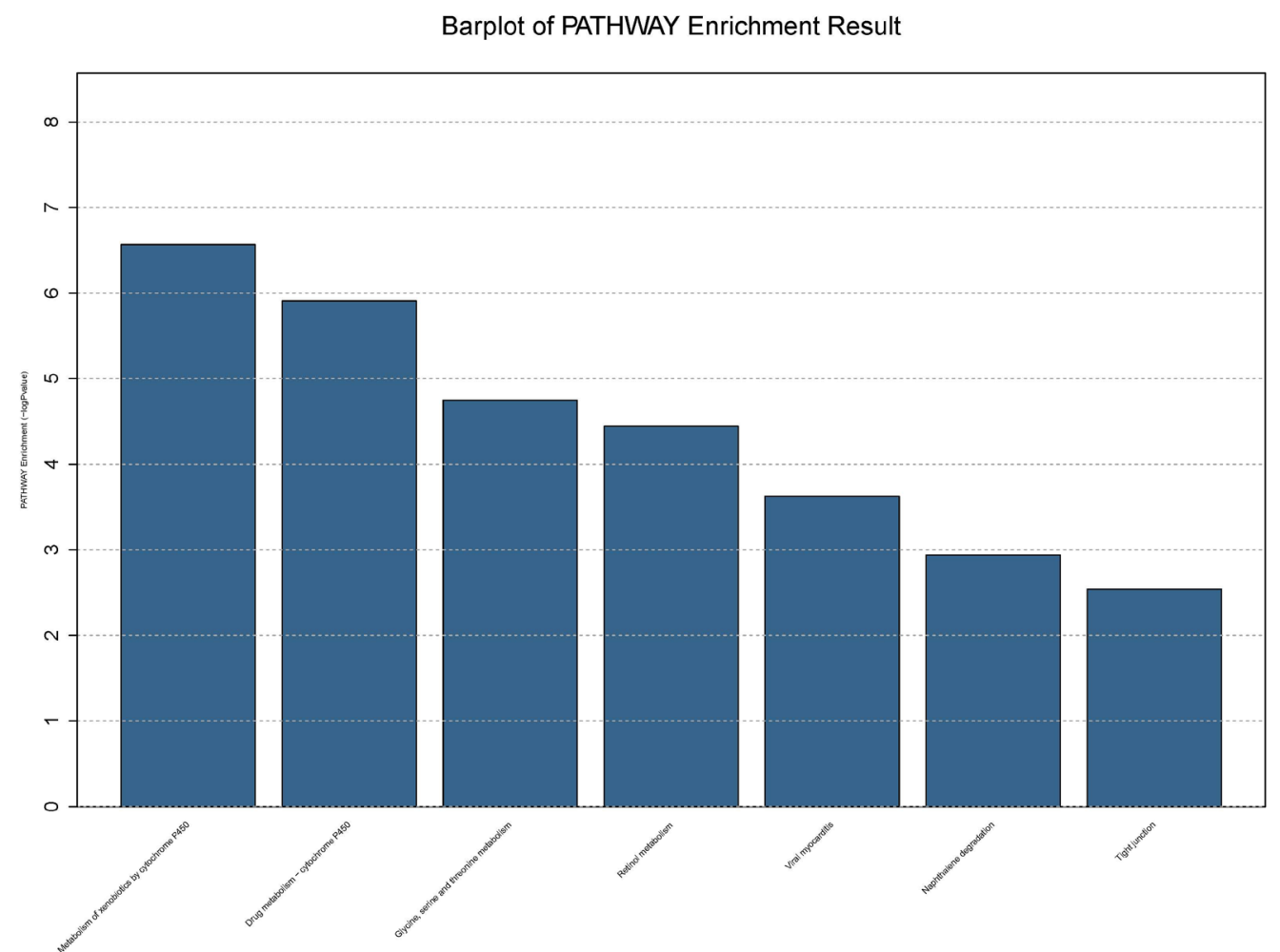

Figure 4. Kyoto Encyclopedia of Genes and Genomes (KEGG) pathway enrichment analysis of differentially expressed genes (DEGs) from ovary and testis of Macrobrachium nipponense.

\section{DISCUSSION}

Reproduction and development were very complicated processes with many unclear genetic regulatory mechanisms. The animal tests and ovary are multifunctional organs that play a key role in reproduction, sexual maturity, and secretion of hormones to regulate growth and development (Chu et al., 2006). Recent studies showed that constructing cDNA libraries and transcriptome profiles provide a rapid method for gene discovery to fill in the gaps in the genome database of M. nipponense (Wu et al., 2009; Qiao et al., 2012; Ma et al., 2012). In the expression profiling of testis and ovary of $M$. nipponense described in this study, the tag-based RNA-Seq approach proved successful for expression profiling, and over 27 million Illumina 
reads were generated with more than 10,000 significant DEGs being identified. Compared to the EST libraries of M. nipponense (Wu et al., 2009; Qiao et al., 2012), which generated 3256 (ovary) and 5202 (testis) ESTs, this approach was more effective and productive with lower cost. Comparing the 2 libraries, ovary DEGs with upregulated expression (54.5\%) were more abundant than testis DEGs with upregulated expression. Of these DEGs, only a small number were annotated in the NCBI database, and novel genes accounted for $69.4 \%$ of the DEGs. These DEGs can provide a large candidate database to mine novel genes that play key roles in reproduction and gonad development in M. nipponense and other crustaceans.

Table 4. Selected genes of interest for reproduction, development, and sex in the Macrobrachium nipponense expression profile.

\begin{tabular}{|c|c|c|c|c|c|}
\hline No. & Description & Up or Down ${ }^{1}$ & Fold $(\mathrm{T} / \mathrm{O})^{*}$ & Accession No. of Hit & E value \\
\hline 1 & Arginine kinase 1 & $\uparrow$ & 23.6 & gi $|307715368|$ & $7.56 \mathrm{E}-05$ \\
\hline 2 & Cathepsin D & $\uparrow$ & 28.1 & gi $|146217392|$ & $7.35 \mathrm{E}-04$ \\
\hline 3 & Chitinase 1 precursor & $\uparrow$ & 34.0 & gi $|195969366|$ & 8.34E-03 \\
\hline 4 & Crustin & $\uparrow$ & 80.8 & gi $|146432699|$ & $1.31 \mathrm{E}-10$ \\
\hline 5 & Cytochrome P450 V20 & $\uparrow$ & 76.4 & gi $|374923109|$ & $1.10 \mathrm{E}-06$ \\
\hline 6 & Cytochrome $\mathrm{c}$ oxidase subunit I & $\uparrow$ & 10.3 & gi $|322422337|$ & $3.15 \mathrm{E}-25$ \\
\hline 7 & Kazal-type serine proteinase inhibitor & $\uparrow$ & 28.4 & gi $|391234179|$ & $8.49 \mathrm{E}-07$ \\
\hline 8 & Male reproduction-related LIM protein & $\uparrow$ & 14.3 & gi $|146432689|$ & $3.73 \mathrm{E}-04$ \\
\hline 9 & Male reproduction-related protein A & $\uparrow$ & 89.0 & gi $|146432625|$ & $3.87 \mathrm{E}-11$ \\
\hline 10 & Male reproduction-related protein B & $\uparrow$ & 77.64 & gi| $146432657 \mid$ & $2.42 \mathrm{E}-10$ \\
\hline 11 & Male reproduction-related protein Mar-Mrr & $\uparrow$ & 1027.3 & gi $|146432671|$ & $5.52 \mathrm{E}-11$ \\
\hline 12 & Sperm gelatinase & $\uparrow$ & 294.3 & gi $|391234181|$ & $7.12 \mathrm{E}-16$ \\
\hline 13 & Spermatogenesis-associated protein 13 & $\uparrow$ & 6.85 & gi $|307179210|$ & $5.15 \mathrm{E}-03$ \\
\hline 14 & Sox 14 protein & $\uparrow$ & 7.83 & gi|3913352| & 0.018 \\
\hline 15 & Serine proteinase inhibitor 8 & $\uparrow$ & 165.1 & gi $|288188858|$ & $1.92 \mathrm{E}-11$ \\
\hline 16 & JHE-like carboxylesterase 1 & $\uparrow$ & 13.9 & gi $|326579691|$ & $9.71 \mathrm{E}-03$ \\
\hline 17 & Juvenile hormone esterase & $\uparrow$ & 9.0 & gi $|157109005|$ & 0.010 \\
\hline 18 & Peritrophin & $\uparrow$ & 42.25 & gi|$|19879412|$ & $4.53 \mathrm{E}-07$ \\
\hline 19 & Heat shock protein 90 & $\uparrow$ & 13.8 & gi|301299151| & $1.24 \mathrm{E}-04$ \\
\hline 20 & Cyclin-L1 & $\uparrow$ & 19.9 & gi|307189308| & $1.34 \mathrm{E}-05$ \\
\hline 21 & Cathepsin L & $\downarrow$ & 0.286 & gi $|330434686|$ & $3.47 \mathrm{E}-03$ \\
\hline 22 & Extra sex combs & $\downarrow$ & 0.384 & gi $|2352418|$ & $1.59 \mathrm{E}-03$ \\
\hline 23 & Gametocyte-specific factor 1 & $\downarrow$ & $3.37 \mathrm{E}-03$ & gi $|225710058|$ & $1.21 \mathrm{E}-07$ \\
\hline 24 & PREDICTED: ubiquitin-conjugating enzyme E2 S-like & $\downarrow$ & 0.128 & gi 383850836 & $1.18 \mathrm{E}-05$ \\
\hline 25 & VASA-like protein & $\downarrow$ & 0.367 & gi $|349806885|$ & 8.01E-03 \\
\hline 26 & Vitellogenin & $\downarrow$ & 7.18E-03 & gi $|16151644|$ & $9.31 \mathrm{E}-03$ \\
\hline 27 & Vitellogenin receptor & $\downarrow$ & 7.98E-03 & gi $|301070472|$ & $1.59 \mathrm{E}-16$ \\
\hline 28 & Kunitz-type protease inhibitor 1 & $\downarrow$ & $5.51 \mathrm{E}-03$ & gi $|405978887|$ & $3.89 \mathrm{E}-15$ \\
\hline 29 & PREDICTED: gonadotropin-releasing hormone receptor-like & $\downarrow$ & 0.013 & gi $|380019458|$ & 5.57E-11 \\
\hline 30 & PREDICTED: oocyte zinc finger protein XICOF7.1-like & $\downarrow$ & 0.237 & gi $|301629236|$ & $1.86 \mathrm{E}-04$ \\
\hline 31 & PREDICTED: oocyte zinc finger protein XICOF6-like & $\downarrow$ & 0.340 & gi|326666714| & $8.74 \mathrm{E}-04$ \\
\hline 32 & Cdc42 homolog & $\downarrow$ & 0.024 & gi $|290562982|$ & $1.47 \mathrm{E}-11$ \\
\hline 33 & Trypsin-like serine proteinase 2 & $\downarrow$ & 0.312 & gi $|229258304|$ & $1.21 \mathrm{E}-04$ \\
\hline 34 & Cathepsin C & $\downarrow$ & 0.192 & gi $|195971120|$ & $1.03 \mathrm{E}-03$ \\
\hline 35 & Broad-complex protein isoform 4 & $\downarrow$ & 0.114 & gi|358442122| & $2.91 \mathrm{E}-06$ \\
\hline $36^{\#}$ & Protein fem-1-like protein & - & - & gi|307214932| & $8.31 \mathrm{E}-11$ \\
\hline $37^{\#}$ & Fem-1 homolog B-like protein & - & - & gi $|375493328|$ & $1.83 \mathrm{E}-04$ \\
\hline $38^{\#}$ & Sex-determining protein fem-1 & - & - & gi $|170058345|$ & $5.62 \mathrm{E}-06$ \\
\hline $39^{\#}$ & Fushi tarazu factor 1 & - & - & gi| $5306097 \mid$ & 0.089 \\
\hline $40^{\#}$ & Estrogen-related receptor & & & gi $|283825466|$ & 0.073 \\
\hline $41^{\#}$ & Ecdysteroid receptor isoform 1 & & & gi|226316403| & 0.074 \\
\hline $42^{\#}$ & Sex-determining region $Y$ protein & & & gi $|326430125|$ & 0.052 \\
\hline
\end{tabular}

${ }^{1}$ An upward arrow indicates upregulation in testis; a downward arrow indicates downregulation in testis (upregulation in ovary). "Gene had no significant differential expression between testis and ovary. *Fold (T/O) means the expression ratio of DEGs between testis and ovary. 


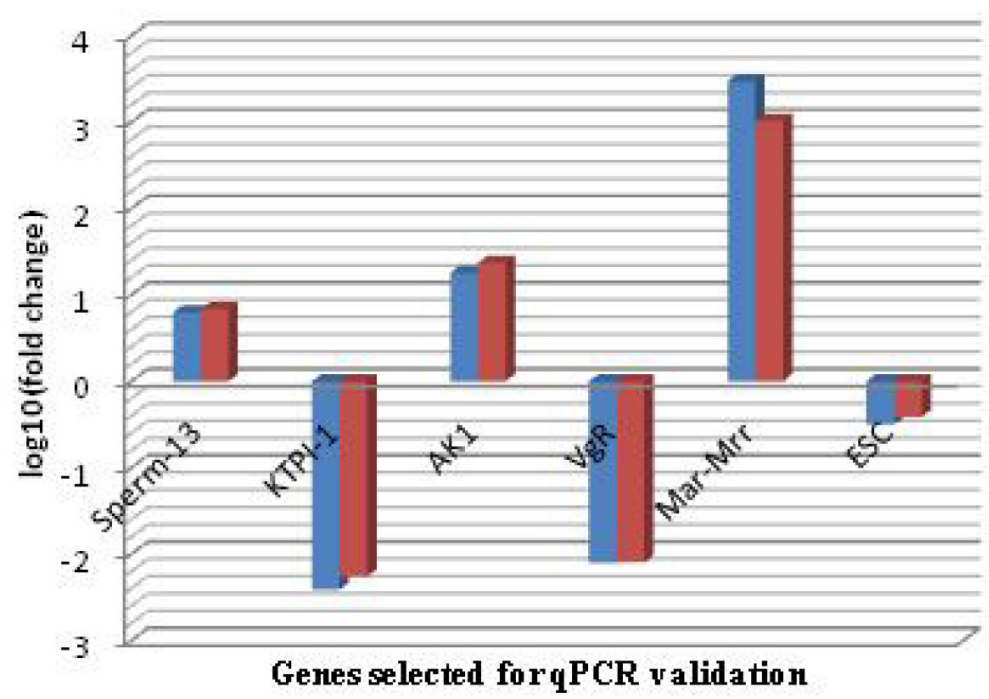

qRT-PCR

Solexa

Figure 5. Six genes randomly selected for quantitative real-time polymerase chain reaction (qRT-PCR) validation. The $\mathrm{x}$-axis indicates the name of genes selected for qRT-PCR validation. The $\mathrm{y}$-axis represents the fold-change of expression between testis and ovary $(\log 10)$. qRT-PCR indicates the data from the qRT-PCR validation; Solexa indicates the data from the expression profile. Sperm-13 = spermatogenesis-associated protein 13; KTPI-1 = kunitztype protease inhibitor 1 ; $\mathrm{AK} 1=$ arginine kinase $1 ; \mathrm{VgR}=$ vitellogenin receptor; Mar-Mrr = male reproductionrelated protein Mar-Mrr; ESC = extra sex comb.

Table 5. Primers used in quantitative real-time polymerase chain reaction (qRT-PCR) validation.

\begin{tabular}{ll}
\hline Name & Primer sequence (5'-3') \\
\hline Sperm-13 & F: TCTCTTTGGATGGCTTCCTT \\
KTPI-1 & R: CGTTCATTGACAAGAAGTGC \\
AK1 & F: ATTGGTGCTCCCTGAGTTTGTAA \\
& R: GCTTGCAATCGTCATCATAGCTT \\
VgR & F: AAGATCAAGGGCAACATCAACGCC \\
Mar-Mrr & R: AGGCGCTCTTCATGAGGTCTCAA \\
ESC & F: AGAGAAATGGGCGAGTACAAGTT \\
& R: CTCGATAGTCACCAAGGAGCTTT \\
& F: ATTCATCCTGGTCTCTGAAGCTG \\
& R: CCCATCCTAGGGCAAATGTATGA \\
& F: TGGCTACCTTTGGTGGAGTAGAG \\
\hline
\end{tabular}

GO analyses classified the unitags from 2 libraries into various terms based on the annotation in the NCBI nr protein database. The terms were similar to those from the reference transcriptomic data of this and other species, indicating that genes encoding these functions may be conserved and easy to annotate in the database. It also provides a comprehensive and structured vocabulary for describing genes in the gonads of M. nipponense. Enrichment analyses were performed for the significant DEGs. The enrichment analysis results support the biological significance of expression profiles. The KEGG enrichment pathway results revealed that significant DEGs were strongly associated with metabolism of xenobiotics by cytochrome P450, and drug metabolism by cytochrome P450. Cytochrome P450 genes are important for the biosynthesis of sex steroids, which have crucial roles in reproduction, including sex differ- 
entiation, gametogenesis, and gamete maturation, in vertebrate gonads and some invertebrates (Callard et al., 2001; Mizuta and Kubokawa, 2007). Actually, many gene tags were related to cytochrome P450 in the expression profiles, which indicated that these genes might have important functions in the reproduction process and gonad development of $M$. nipponense. The predicted metabolic pathways, together with the GO analysis, are useful for further investigations of gene function.

In this research, 10,018 DEGs involved in reproduction in M. nipponense and other potential candidates were identified. Of these, $69.4 \%$ were new without any annotation and require further investigation. The other DEGs with annotations were divided into 2 groups, those that were upregulated in testis and those that were upregulated in ovary. Without considering the conserved housekeeping genes, many important reproduction-related genes were discovered.

Vitellogenin, which is a good indicator of female ovarian activity, has been proposed to be synthesized in the ovary and hepatopancreas in decapod crustaceans (Tsukimura, 2001). It was reported to be expressed in both the hepatopancreas and ovaries in many prawns, such as Penaeus vannamei, P. semisulcatus, and P. monodon, but it is only expressed in the female hepatopancreas in M. rosenbergii (Avarre et al., 2003; Tiu et al., 2006). It was also not identified in the ovary cDNA library (Wu et al., 2009). In this study, vitellogenin and vitellogenin receptor were found to be expressed at a moderate level in ovary of $M$. nipponense. Whether vitellogenin mRNAs exit the mature ovary, hepatopancreas, or both is not clear and requires further studies.

Furthermore, many other reproduction-related genes were also discovered in the profile. Cathepsin $\mathrm{L}$ has activities that have been associated with yolk processing during vitellogenesis in rainbow trout (Kwon et al., 2001). Cathepsin C was found to be involved in the final stages of oocyte maturation in the kuruma prawn Marsupenaeus japonicus (Qiu et al., 2008a). These cathepsins also presented significantly upregulated expression in ovary in this paper, which is consistent with the previous reports. Cathepsin D, which was found to have the greatest tissue-specific immunoexpression in the testis in rat male reproductive organs (Burdan et al., 2006), displayed significantly upregulated expression in testis in this study. Male reproductive-related proteins such as kazal-type serine proteinase inhibitor, sperm gelatinase, male reproductive-related LIM protein, male reproductive-related protein Mar-Mrr, and male reproductive-related protein A and B showed strong expression in testis. These proteins were proven to be male-specific proteins in M. rosenbergii (Dai et al., 2009). The functions of these proteins in $M$. nipponense remain to be determined. Gonadotropin-releasing hormone, which has been demonstrated by immunocytochemistry in $M$. rosenbergii and $P$. monodon to be located in the pre-vitellogenic and early vitellogenic oocytes and mature ovaries, was also found to display high expression in ovary of $M$. nipponense, suggesting a specific role in ovarian maturation in this prawn (Ngernsoungnern et al., 2008a,b). The extra sex comb gene, which had significantly higher expression in ovary than in testis in this paper, plays important roles in determining segment identity (Struhl, 1981). Broad-complex isoform 4 was identified in the profile and showed significant high expression in ovary. It contributes to ovarian development in P. monodon (Buaklin et al., 2013). Many other reproduction- or development-related factors, such as gametocyte-specific factor 1, vitelline membrane outer layer 1-like protein, and spermatogenesis-associated protein 13, displayed relative upregulated expression in ovary and testis. Characterization of these genes with significant differences in expression levels in oriental river prawn may lead to great advances in our understanding of the molecular mecha- 
nism of reproduction.

In this study, we also identified putative genes related to reproduction and sex determination with no significant difference in expression levels in M. nipponense. Recent studies indicate that ecdysteroids, which are also considered as molting hormones, play a major role in regulating vitellogenesis, ovarian maturation, and protein synthesis in decapods (Brown et al., 2009). A high titer of estrogens was found during vitellogenic stages, suggesting a possible role in the stimulation of vitellogenesis (Fairs et al., 1990). We found estrogen-related receptor, ecdysteroid receptor, and ecdysteroid regulated-like protein homologs in our expression profile. Besides, we identified other putative genes related to sex determination, such as Fushi tarazu factor 1, sex-determining protein fem-1, and sex-determining region Y protein. Fushi tarazu factor 1, which was originally found as a regulator of the Drosophila homeobox segmentation gene FTZ, is an essential factor in sex differentiation (Lavorgna et al., 1991). The sex-determining protein complex of FEM-1/FEM-2/FEM-3 promotes male development by inhibiting the activity of the terminal sex-determination factor TRA-1A, a zinc-finger transcription factor that promotes hermaphrodite development by repressing the expression of genes required for sperm production and somatic male development (Mapes et al., 2010). Sex-determining region $\mathrm{Y}$ protein acts dominantly to trigger differentiation of testes from undifferentiated gonads (Berta et al., 1990; Koopman et al., 1991). However, these important genes showed no significant difference in expression levels in this study. The distribution and function of these genes in M. nipponense require further study.

In conclusion, our study constructed a database from an RNA-Seq project to identify genes with significant differences in expression level between the mature testis and ovary of $M$. nipponense. One of the most important aspects of this study is the association of sequences and related expression information with reproduction functions. These annotations will advance research in the field of molecular genetics in M. nipponense and offer a valuable resource for further research related to reproduction in crustaceans.

\section{ACKNOWLEDGMENTS}

Research supported by the Jiangsu Provincial Natural Science Foundation for Young Scholars of China (Grant \#BK2012091), the National Natural Science Foundation of China (Grant \#31272654), the National Science \& Technology Supporting Program of the 12th Fiveyear Plan of China (Grant \#2012BAD26B04), the Science \& Technology Supporting Program of Jiangsu Province (Grant \#BE2012334), and the Basic R\&D Fund for National Research Institutions of China (\#2013JBFM15).

\section{REFERENCES}

Audic S and Claverie JM (1997). The significance of digital gene expression profiles. Genome Res. 7: 986-995.

Avarre JC, Michelis R, Tietz A and Lubzens E (2003). Relationship between vitellogenin and vitellin in a marine shrimp (Penaeus semisulcatus) and molecular characterization of vitellogenin complementary DNAs. Biol. Reprod. 69: 355-364.

Berta P, Hawkins JR, Sinclair AH, Taylor A, et al. (1990). Genetic evidence equating SRY and the testis-determining factor. Nature 348: 448-450.

Brown MR, Sieglaff DH and Rees HH (2009). Gonadal ecdysteroidogenesis in arthropoda: occurrence and regulation. Annu. Rev. Entomol. 54: 105-125.

Buaklin A, Sittikankaew K, Khamnamtong B, Menasveta P, et al. (2013). Characterization and expression analysis of the 
Broad-complex (Br-c) gene of the giant tiger shrimp Penaeus monodon. Comp. Biochem. Physiol. B Biochem. Mol. Biol. 164: 280-289.

Burdan F, Szumilo J, Dudka J, Dabrowski A, et al. (2006). The activity and immunoexpression of cathepsin D in rat male reproductive organs. Folia Morphol. 65: 111-115.

Bureau of Fishery MoAPRC (2011). Fisheries Economic Statistics. In: China Fishery Yearbook China Agricultural Press, Beijing, 236.

Cai Y and Ng PKL (2002). The freshwater palaemonid prawns (Crustacea: Decapoda: Caridea) of Myanmar. Hydrobiologia 487: 59-83.

Callard GV, Tchoudakova AV, Kishida M and Wood E (2001). Differential tissue distribution, developmental programming, estrogen regulation and promoter characteristics of cyp19 genes in teleost fish. J. Steroid Biochem. Mol. Biol. 79: 305-314.

Chu SL, Weng CF, Hsiao CD and Hwang PP (2006). Profile analysis of expressed sequence tags derived from the ovary of tilapia, Oreochromis mossambicus. Aquaculture 251: 537-548.

Dai ZM, Zhu XJ and Yang WJ (2009). Full-length normalization subtractive hybridization: a novel method for generating differentially expressed cDNAs. Mol. Biotechnol. 43: 257-263.

Fairs NJ, Quinlan PT and Goad LJ (1990). Changes in ovarian unconjugated and conjugated steroid titers during vitellogenesis in Penaeus monodon. Aquaculture 89: 83-99.

Koopman P, Gubbay J, Vivian N, Goodfellow P, et al. (1991). Male development of chromosomally female mice transgenic for Sry. Nature 351: 117-121.

Krungkasem C, Ohira T, Yang WJ, Abdullah R, et al. (2002). Identification of two distinct molt-inhibiting hormonerelated peptides from the giant tiger prawn Penaeus monodon. Mar. Biotechnol. 4: 132-140.

Kwon JY, Prat F, Randall C and Tyler CR (2001). Molecular characterization of putative yolk processing enzymes and their expression during oogenesis and embryogenesis in rainbow trout (Oncorhynchus mykiss). Biol. Reprod. 65: 1701-1709.

Lavorgna G, Ueda H, Clos J and Wu C (1991). FTZ-F1, a steroid hormone receptor-like protein implicated in the activation of fushi tarazu. Science 252: 848-851.

Livak KJ and Schmittgen TD (2001). Analysis of relative gene expression data using real-time quantitative PCR and the 2[-Delta Delta C(T)] Method. Methods 25: 402-408.

Ma K, Qiu G, Feng J and Li J (2012). Transcriptome analysis of the oriental river prawn, Macrobrachium nipponense using 454 pyrosequencing for discovery of genes and markers. PLoS One 7: e39727.

Mapes J, Chen JT, Yu JS and Xue D (2010). Somatic sex determination in Caenorhabditis elegans is modulated by SUP26 repression of tra-2 translation. Proc. Natl. Acad. Sci. U. S. A. 107: 18022-18027.

Marioni JC, Mason CE, Mane SM, Stephens M, et al. (2008). RNA-seq: an assessment of technical reproducibility and comparison with gene expression arrays. Genome Res. 18: 1509-1517.

Mizuta T and Kubokawa K (2007). Presence of sex steroids and cytochrome P450 genes in amphioxus. Endocrinology 148: 3554-3565.

Mortazavi A, Williams BA, McCue K, Schaeffer L, et al. (2008). Mapping and quantifying mammalian transcriptomes by RNA-Seq. Nat. Methods 5: 621-628.

Ngernsoungnern A, Ngernsoungnern P, Kavanaugh S, Sower SA, et al. (2008a). The identification and distribution of gonadotropin-releasing hormone-like peptides in the central nervous system and ovary of the giant freshwater prawn, Macrobrachium rosenbergii. Invert. Neurosci. 8: 49-57.

Ngernsoungnern P, Ngernsoungnern A, Kavanaugh S, Sobhon P, et al. (2008b). The presence and distribution of gonadotropin-releasing hormone-liked factor in the central nervous system of the black tiger shrimp, Penaeus monodon. Gen. Comp. Endocrinol. 155: 613-622.

Olmstead AW and Leblanc GA (2002). Juvenoid hormone methyl farnesoate is a sex determinant in the crustacean Daphnia magna. J. Exp. Zool. 293: 736-739.

Pascual C, Valera E, Re-Regis C and Gaxiola G (1998). Effect of water temperature on reproductive tract condition of Penaeus setiferus adult males. J. World Aquacult. Soc. 29: 477-484.

Qian YQ, Li Y, Yang F, Yu YQ, et al. (2012). Two Kazal-type protease inhibitors from Macrobrachium nipponense and Eriocheir sinensis: comparative analysis of structure and activities. Fish Shellfish Immunol. 32: 446-458.

Qiao H, Fu H, Jin S, Wu Y, et al. (2012). Constructing and random sequencing analysis of normalized cDNA library of testis tissue from oriental river prawn (Macrobrachium nipponense). Comp. Biochem. Physiol. Part D. Genomics Proteomics 7: 268-276.

Qiu L, Jiang S, Huang J, Wang W, et al. (2008a). Molecular cloning and mRNA expression of cathepsin C gene in black tiger shrimp (Penaeus monodon). Comp. Biochem. Physiol. A Mol. Integr. Physiol. 150: 320-325.

Qiu L, Jiang S, Zhou F, Zhang D, et al. (2008b). Molecular cloning of the black tiger shrimp (Penaeus monodon) elongation 
factor 2 (EF-2): sequence analysis and its expression on the ovarian maturation stage. Mol. Biol. Rep. 35: 431-438.

Sagi A, Cohen D and Milner Y (1990). Effect of androgenic gland ablation on morphotypic differentiation and sexual characteristics of male freshwater prawns, Macrobrachium rosenbergii. Gen. Comp. Endocrinol. 77: 15-22.

Sasser EW and Singhas CA (1992). Presence of an estriol-like steroid in the blue crab, Callinectes sapidus. Aquaculture 104: 367-373.

Somka Y, Milner Y and Laufer H (1993). A protein synthesis in the ovary of Macrobrachium rosenbergii during the reproductive cycle: effects of methyl fanesoate (MF). Am. Zool. 33: 123.

Struhl G (1981). A gene product required for correct initiation of segmental determination in Drosophila. Nature 293: $36-41$.

Tiu SHK, Hui JHL, Mak ASC and He JG (2006). Equal contribution of hepatopancreas and ovary to the production of vitellogenin (PmVg1) transcripts in the tiger shrimp, Penaeus monodon. Aquaculture 254: 666-674.

Treerattrakool S, Panyim S, Chan SM, Withyachumnarnkul B, et al. (2008). Molecular characterization of gonad-inhibiting hormone of Penaeus monodon and elucidation of its inhibitory role in vitellogenin expression by RNA interference. FEBS J 275: 970-980.

Tsukimura B (2001). Crustacean vitellogenesis: its role in oocyte development. Am. Zool. 41: 465-476.

Wainwright G, Webster SG, Wilkinson MC, Chung JS, et al. (1996). Structure and significance of mandibular organinhibiting hormone in the crab, Cancer pagurus. Involvement in multihormonal regulation of growth and reproduction. J. Biol. Chem. 271: 12749-12754.

Wu P, Qi D, Chen L, Zhang H, et al. (2009). Gene discovery from an ovary cDNA library of oriental river prawn Macrobrachium nipponense by ESTs annotation. Comp. Biochem. Physiol. Part. D. Genomics Proteomics 4: 111-120.

Xie Y, Li F, Wang B, Li S, et al. (2010). Screening of genes related to ovary development in Chinese shrimp Fenneropenaeus chinensis by suppression subtractive hybridization. Comp. Biochem. Physiol. Part. D. Genomics Proteomics 5: 98-104. 